\title{
Julio Cortázar oder die Auflösung der Unilinearität des Buches
}

Wir hatten im bisherigen Verlauf unserer Vorlesung gesehen, dass die Linearität des Schreibens und die Unilinearität des Buches bereits für die historischen Avantgarden ein Ärgernis und mehr noch eine künstlerisch-literarische Herausforderung bildeten, an der sich auch die von den Avantgarden inspirierten Schriftsteller ein ums andere Mal abarbeiteten. Es kann an dieser Stelle nicht darum gehen, die historische Entwicklung all jener Lösungsversuche aufzuarbeiten, welche in langen Jahrhunderten immer wieder erarbeitet wurden und in deren Geschichte die Namen etwa eines Alexander von Humboldt oder eines Fernando Ortiz - auf den ich noch kurz zurückkommen werde - nicht fehlen dürfen. Die oszillierende Bewegung zwischen verschiedenen Kapiteln und Textteilen wie vor allem auch die Infragestellung der Linearität des Buches wurde in großer Radikalität aber von einem Autor betrieben, den ich Ihnen jetzt vorstellen möchte.

Es handelt sich dabei um einen argentinischen Schriftsteller, mit dem wir wieder auf jene Seite des Atlantiks zurückkehren, auf welcher Jorge Luis Borges von Buenos Aires aus wichtige Impulse für die Entwicklung der Literatur wie für die Ausgestaltung der Literaturen der Welt setzte. Doch wäre es ungerecht, einen Julio Cortázar in den Schatten von Jorge Luis Borges zu stellen. Denn dieser außergewöhnlich kreative Schriftsteller entfaltete mit seiner literarischen Praxis wie mit seiner theoriefreudigen Essayistik doch eine so eigenständige Position, welche zwar mit Borges in einigen Punkten übereinstimmte, in anderen aber ganz grundlegend von Überlegungen und Stellungnahmen seines Landsmannes abwich, so dass er heute als eine der ganz großen Gestalten der lateinamerikanischen Literaturen gelten darf.

Ich möchte Ihnen aus dem riesigen Gesamtwerk des Argentiniers an erster Stelle den Roman Rayuela vorstellen, der für uns in vielfacher Weise vorbildgebend ist und zugleich die literarische Entwicklung in einer Richtung vorantrieb, wie sie für unsere Perspektivik in der Tat entscheidend ist. Denn es handelt sich um einen Roman, der die beiden Seiten des Atlantiks miteinander zu verbinden sucht und auf diese Weise eine neue Welt der Literatur in einem Zwischenraum und mehr noch in einem Bewegungsraum schafft, der in seiner Ausgestaltung poetologisch - und ich würde auch sagen epistemologisch - modellbildend wirkte. Zugleich entfaltet er durch die Vielzahl intertextueller Relationen einen literarischen Raum, der vergleichbar ist mit den „Anales“ in Max Aubs gefälschter Künstlerbiographie Jusep Torres Campalans.

Dort hatten die Lagererfahrung und mehr noch das Exil eine wichtige Rolle bei der Herstellung dieses komplexen und transatlantisch breit gefächerten 
Raumes gespielt. Und auch bei Julio Cortázar lässt sich eine Verbindung herstellen zwischen den Grundstrukturen seines Romans und seinen existenziellen Erlebnissen und Erfahrungen, welche ihn aus Protest gegen das Perón-Regime in Argentinien zu Beginn der fünfziger Jahre das Weite - und das hieß damals noch immer Europa und vor allem Paris - suchen ließen. Es ist daher sinnvoll, an den Anfang unserer notwendig kurzen Auseinandersetzung mit diesem wichtigen argentinischen Autor eine biographische Skizze zu stellen, welche uns den Zugang zu seinem Gesamtwerk etwas erleichtern soll.

Julio Cortázar wurde am 26. August 1914 in Brüssel als Sohn des dortigen argentinischen Konsuls geboren und starb am 12. Februar 1984 in seiner Wahlheimat Paris. Wegen der Verhältnisse während des Ersten Weltkriegs wuchs er zunächst in der Schweiz, später dann in Barcelona auf, bevor die Familie 1918 nach Argentinien zurückkehrte. Es ist eigenartig, dass sich zu Beginn dieses Lebenslaufs doch einige Parallelen zu Jorge Luis Borges ergeben, der gleichwohl deutlich älter war, sich aber auch mitten in der „Grande Guerre“ in Europa aufhielt, insbesondere in der Schweiz und Spanien.

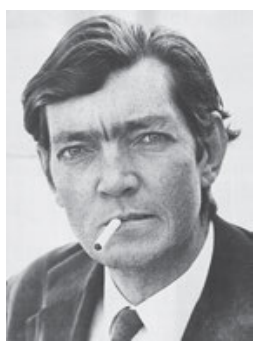

Abb. 102: Julio Cortázar (Brüssel, 1914 - Paris, 1984).

Da der Vater bald schon die Familie verließ, wuchs Cortázar - umgeben von Frauen - in einer Vorstadt von Buenos Aires unter schwierigen finanziellen Bedingungen auf, da es Mutter und Großmutter kaum gelang, Julio und seine ältere Schwester über Wasser zu halten. Früh schon flüchtete sich der Junge in die Welt der Fiktionen und verschlang alles, was ihm unter die Augen kam. Nach einer kurzen Ausbildung an der Universidad de Buenos Aires wurde er 1937 Grundschullehrer in der argentinischen Provinz und später 1944 Universitätsdozent für französische Literatur im argentinischen Mendoza. Nach seiner Entlassung unter der ersten Regierung von Juan Perón verließ er aus politischen Gründen, aber mit einem Schriftstellerstipendium in der Tasche Argentinien, nachdem er sich einige Jahre als Übersetzer durchgeschlagen hatte.

Nach seiner Übersiedlung nach Paris arbeitete Cortázar lange Jahre als freier Übersetzer für die UNESCO und verschiedene internationale Organisationen und brillierte mit seinen Übersetzungen etwa des Robinson Crusoe oder Texten von 
Edgar Allan Poe, der sein eigenes schriftstellerisches Werk stark beeinflusste. Zugleich aber entstanden erste Erzählungen, die den Schriftsteller berühmt machen sollten. Zahlreiche Reisen führten ihn von Paris aus nach Lateinamerika, darunter 1963 eine erste Reise nach Kuba, dem Cortázar zeit seines Lebens eine kritische Treue hielt. Er trat seit 1967 öffentlich für die Kubanische Revolution ein, führte eine berühmte Kontroverse mit dem peruanischen Schriftsteller Mario Vargas Llosa, trat für den Sozialismus ein, aber kritisierte zugleich die stalinistischen Schauprozesse gegen Schriftsteller in Kuba, was ihm viel Häme einbrachte.

Unbeirrt hielt er trotz aller Gegenangriffe von konservativer wie linker Seite an seinen Überzeugungen fest und verteidigte nicht nur den Sozialismus Salvador Allendes, sondern später auch die sandinistische Nikaraguanische Revolution. Er engagierte sich wiederholt politisch für Menschenrechte, war Mitglied des Russell-Tribunals und klagte die Militärregierungen in Argentinien, Uruguay und Chile öffentlichkeitswirksam an. Unmittelbar nach dem Amtsantritt François Mitterrands wurde der überwiegend in Paris lebende Schriftsteller 1981 nach langer politischer Kontroverse endlich in Frankreich eingebürgert. Nach Ende der argentinischen Militärdiktatur führte ihn 1983 eine letzte Reise nach Argentinien. Cortázar blieb sein Leben lang seinen politischen und ethischen Überzeugungen treu, auch wenn er sich damit oftmals in einem Zwischenbereich bewegte und die Kritik aller Seiten auf sich zog.

Man könnte durchaus formulieren, dass sich der streitbare, aber stets humorvolle Argentinier auch im Literarischen innerhalb eines Zwischenbereichs bewegte, den die beiden Seiten des Atlantiks bildeten. Seine intensive literarische Arbeit schlug sich in einer Vielzahl von Erzählungen und Romanen, aber auch literarischen wie literaturtheoretischen Essays nieder. Dabei avancierte er $\mathrm{zu}$ einem der herausragenden Vertreter der fantastischen Literatur, wobei er besonderen Wert darauf legte, in seinem Rückgriff auf die historischen Avantgarden stets nur ein wenig Ingredienzien des französischen Surrealismus in seine Prosa eingestreut zu haben. Bei seiner ästhetischen Ausgestaltung des Fantastischen gab es sicherlich die zahlreichsten Berührungspunkte mit dem literarischen Werk des Jorge Luis Borges, zu dem er politisch freilich in klarer Opposition stand.

In seinen meisterhaften Erzählungen dominiert ein spielerischer, aber stets humorvoller Ton, wobei sich in seinem Schreiben das Alltägliche mit dem Fantastischen, das Faktische mit dem Fiktionalen so mischt, dass die Grenzen zwischen Realität und Imagination völlig verschwimmen. Literarisch war Cortázar auf beiden Seiten des Atlantiks, besonders aber in der französischen Literatur und Literaturtheorie zu Hause. Seine poetologisch-literarästhetischen Einsichten wie etwa die Bestimmung der Erzählung als eines narrativen Pfeiles, der auf direktem Wege ins Schwarze treffen müsse, haben die Diskussionen um die Modellhaftigkeit der lateinamerikanischen Literaturen über lange Jahrzehnte befeuert. 
Julio Cortázar ist sicherlich einer der spannendsten und zugleich aufschlussreichsten Autoren, um die Zerrissenheit und Gespaltenheit der lateinamerikanischen Literaturen und der Literaturen der Postmoderne überhaupt zwischen den Erfordernissen politischer Ansprüche oder Werte einerseits und jenen ästhetischer Ausdrucksformen andererseits herauszuarbeiten. Eine Zerrissenheit, die Julio Cortázar über ein langes Leben hin beschäftigt und teilweise in Mitleidenschaft gezogen, aber auch zu einer Vielzahl kreativer Antworten im diktionalen wie fiktionalen Bereich gebracht hat. Kaum einer hat so intensiv wie er die Höhen und die Tiefen politischen Engagements als Schriftsteller mitgemacht. Sie bemerken vielleicht, wieviel Bewunderung in meinen Worten steckt!

Cortázars Mühen waren Mühen in einem auch politischen Zwischenbereich. Denken wir nur an seine Positionierungen bezüglich der Kubanischen Revolution, für die er in den sechziger Jahren eintrat, die er aber im Gefolge der sogenannten Padilla-Affäre in einem hochinteressanten Text mit einer „solidarischen Kritik“ (wie er sich damals ausdrückte) bedachte, die man ihm von Seiten der kubanischen Führung und ihrer Intellektuellen über lange Jahre vorwarf. Von einem anerkannten und geliebten Mitstreiter der Revolution, der in vielen Jurys vertreten und auch andernorts gehört war, verwandelte er sich innerhalb kürzester Zeit in eine Persona non grata, wie sie damals häufig ins Kreuzfeuer kubanischer Intellektuellengeschosse gerieten. Plötzlich war er nun kein gerngesehener Gast mehr in der Casa de las Américas, in deren damals noch bedeutender Zeitschrift er große und wichtige Texte veröffentlicht hatte. Es sollte bis zu seinem Tod im Jahr 1984 dauern, bis die Zeitschrift nach erprobtem Muster den nunmehr Schweigenden wieder in die Gemeinschaft der Kubanischen Revolution mitaufnahm und ihm, dem ,lieben Julio‘, eine Sondernummer der Casa de las Américas widmete. Cortázar war nicht immer geschickt und diplomatisch in seinen Äußerungen, aber doch stets redlich und in seinem Kampf um Menschenrechte unbeirrbar.

All dies soll uns heute aus Zeitgründen jedoch nur mehr am Rande beschäftigen, obwohl seine Positionierung im politischen Feld lateinamerikanischer Intellektueller auch für die Entwicklung seiner ästhetischen Positionen von Bedeutung wäre! Würden wir seinen Roman Libro de Manuel im Rahmen unserer Vorlesung $\mathrm{zu}$ besprechen haben, so ginge ich ausführlicher auf all diese Fragen ein. Der Kontext unserer Literaturen im Zeichen der Postmoderne führt uns aber innerhalb seines erzählerischen Gesamtwerkes zu seinem wohl bekanntesten Prosatext überhaupt, nämlich dem 1963 erstmals veröffentlichten Roman Rayuela, der international Furore machte und sicherlich für die Auslösung des sogenannten „Boom“ der lateinamerikanischen Literaturen mitverantwortlich zu machen ist. Ich möchte der allzu kurzen Analyse dieses großartigen Romans die folgenden Seiten widmen. 
Der Roman setzt mit einer Textpassage ein, welche als „Tablero de dirección“ bezeichnet wird. Darin erfährt die Leserschaft, dass das Buch, das sie in ihren Händen hält, eigentlich mehrere Bücher sind, zumindest aber zweie. Das geneigte Lesepublikum wird sofort zu einer Wahl gezwungen, nämlich eines der beiden vom Autor angebotenen Bücher auszuwählen. Welche sind dies? Und wie kann ein Buch zwei oder gleich mehrere Bücher sein?

Zum einen wird dem Leser vorgeschlagen, den Band auf ganz traditionelle Weise linear zu lesen und dabei bis zum Kapitel 56 vorzudringen, an dessen Ende drei Sternchen für das Wörtchen „Fin“ stehen. Hört sich zunächst einmal vernünftig an! Der Leser, und das ist dann schon etwas überraschender, könne dann getrost und ohne Gewissensbisse auf die nachfolgenden Kapitel verzichten, seien diese Kapitel doch - und wir denken natürlich auch an Max Aubs unverzichtbaren Prolog - verzichtbare Kapitel, von deren Lektüre ohne Probleme abgesehen werden könne. Nun, das Buch ist sicherlich dick genug, als dass die ersten 56 Kapitel einen vollständigen Roman bilden könnten. Aber schließlich hat man ja den ganzen Band bezahlt, und allein aus diesem Grunde würde es einen schon interessieren, auch den ,Rest‘ des Buches kennenzulernen.

Die zweite Lektüreweise ist nun diese: Das Lesepublikum folgt einer genau angegebenen Wegstrecke durch die insgesamt 155 Kapitel, von denen nun nicht 99 ausgespart bleiben, sondern alle gelesen werden sollen, so dass es bei dieser zweiten Lektüreweise keine verzichtbaren Seiten im Buch gibt. Das ist schon mal eine gute Nachricht für sparsame Leserinnen und Leser! Doch die Leserschaft solle nicht einer linearen Abfolge durchnummerierter Kapitel, sondern einem Wegeplan folgen, welcher ständige Sprünge zwischen den drei den Roman bildenden Teilen vorsieht. Dieser Parcours fängt also mit Kapitel 73 an, um dann mit Kapitel 1 weiterzugehen, springt wieder weiter zu einem späteren Kapitel und so fort: Es ist wie ein Hüpfspiel, und eben das ist die Metaphorik von Rayuela. Zurecht wurde der Roman auch ins Deutsche übersetzt unter dem Titel Himmel und Hölle.

Auf diese Weise entsteht - und dies ist charakteristisch für Julio Cortázar, sein Leben und Schaffen - ein wunderbarer Bewegungsraum im Zwischenraum, innerhalb dessen die Protagonisten wie auch die Leserinnen und Leser in ständiger Bewegung sind. Es ergibt sich ein diskontinuierliches Hin- und Herspringen im Buch selbst, das zugleich auch ein Springen zwischen Alter und Neuer Welt ist. Die Leserschaft hüpft zwischen der Welt Oliveiras und der Maga im „Club de la Serpiente“ in Paris einerseits, der Welt Oliveiras in Buenos Aires, später im Irrenhaus mit seinen Freunden Traveler und (auch hier eine Dreiecksbeziehung) Talita andererseits. Schließlich wird der dritte Teil mit seinen zum Teil sehr kurzen Kapiteln letztlich Morelli, einem Schriftsteller und Alter Ego Cortázars, zugeschrieben. Er enthält eine Vielzahl von Zitaten, die durchaus auch in Max 
Aubs Roman hätten einbezogen werden können. Es bildet sich ein Wege- und ein Lektüremuster heraus, welches der Linearität von Schreiben und Lesen diametral entgegengesetzt ist.

In diesem Bewegungsspiel zwischen den beiden Seiten des Atlantiks finden wir auch ein kurzes Kapitel, Nummer 128, in dem nur ein Zitat von Antonin Artaud steht, jenes von Breton ausgebürgerten Surrealisten und Schöpfers des Théâtre de la cruauté, den wir als Zivilisationsflüchtling aus Europa, der im mexikanischen Traum Zuflucht zu finden hoffte, bereits mehrfach kennengelernt hatten. Da ich versuchen möchte, die Parallelen zu Jusep Torres Campalans ganz nebenbei miteinzubauen, sollten wir uns dieses kurze Zitat aus Antonin Artauds Text einmal genauer anschauen:

„Wir sind so einige in dieser Epoche, die gegen die Dinge vorgehen und in uns Räume für das Leben schaffen wollten, Räume, die nicht existierten und die keinen Raum zu finden schienen. “ ARTAUD, Le Pèse-nerfs. ${ }^{1}$

Julio Cortázar beruft sich mit dem Einbau dieses Kapitels auf einen Avantgardisten, einen dissidenten französischen Surrealisten, der die Trennung zwischen Kunst und Leben mit allem, was ihm zur Verfügung stand, bekämpfte und für den die Welt Amerikas zumindest zeitweise eine Realisierung seines Lebenstraumes $\mathrm{zu}$ versprechen schien. Es geht in diesem Zitat um die Schaffung eines weiteren Raumes für das Leben, um Umgestaltung und neue Konzeption eines Lebens, das nicht länger der Macht der Dinge und der faktischen Ding-Welt zu folgen genötigt war. Wir können vieles von dieser Problematik des Raums auch in den Romanen Max Aubs und Julio Cortázars, aber auch in den beiden in ihnen enthaltenen Künstlerfiguren Jusep Torres Campalans und Morelli finden. Denn letztlich sind beide Romane von Aub wie Cortázar dank ihres experimentellen Charakters darauf ausgelegt, den Raum des Romans, den der Kunst und der Wahrnehmung beziehungsweise des Schreibens und Malens wesentlich zu erweitern. Mit dieser Erweiterung des Raumes der Kunst sollte zugleich eine Erweiterung des Raumes des Lebens erfolgen, eine Vorstellung, welche beide Künstler mit den historischen Avantgarden verband.

Gleichzeitig sollte mit beiden Romanen und für beide Künstler eine Veränderung des geokulturellen Raumes verbunden sein, welche die Gleichstellung des amerikanischen (Literatur-) Raumes auf Augenhöhe mit dem Raum der europäischen Literaturen beinhaltete. Wir können aus dieser Perspektive ohne jeden

1 Cortázar, Julio: Rayuela. Edición crítica coordinada por Julio Ortega y Saúl Yurkievich. São Paulo: EdUSP 1996, Kap. 128, S. 412. 
Zweifel feststellen, dass eine geokulturelle Raumerweiterung bei den Literaturen im Zeichen der Postmoderne hinzutritt. Es handelt sich um eine grundlegende Raumerweiterung, die sich in den fünfziger und beginnenden sechziger Jahren bereits Bahn zu brechen begann, um dann in den siebziger Jahren vor einem stabiler gewordenen theoretischen Hintergrund einerseits und einer veränderten kulturellen und massenkulturellen Situation andererseits zu einem Topos und zu einer Tatsache vor allem transatlantischer Literaturbeziehungen zu werden.

Gewiss: In den fünfziger und sechziger Jahren, also zum Zeitpunkt der Veröffentlichung von Jusep Torres Campalans wie von Rayuela, war dieser geokulturelle Dominanten-Wechsel mit der damit einhergehenden Veränderung oder gar Beseitigung der Asymmetrie literarischer Beziehungen zwischen Alter und Neuer Welt noch eine ferne Utopie. Ich darf aus unserer Perspektive zu Beginn des dritten Jahrzehnts des 21. Jahrhunderts hinzufügen, dass es wichtige Schritte in Richtung Einlösung dieser Utopie gab, dass die Utopie symmetrischer literarischer Relationen bis heute jedoch nicht verwirklicht ist. Immerhin: Es zeigten sich damals in der avancierten literarischen Praxis die Zeichen einer Transformation des Systems einer zentrierten Weltliteratur hin zu einem viellogischen System der Literaturen der Welt.

Die grundlegende Problematik von Julio Cortázars Rayuela erfasst aber nicht nur die Poetik literarischer Schöpfung, sondern auch die Legetik und damit nicht zuletzt die Position der Lesenden. Nicht umsonst konnte ich bei Max Aub in Jusep Torres Campalans auf bestimmte negative Verbindungen von empfangender Weiblichkeit und männlicher Schöpferkraft beziehungsweise zwischen Männlichkeit und Produktivität im Gegensatz zu weiblicher Passivität aufmerksam machen. Bitte vergessen Sie nicht, dass wir uns zu Beginn der sechziger Jahre befinden und dass gerade auch jene Strömungen, die von den historischen Avantgarden ausgingen, von Beginn - und damit von den Futuristen - an ganz im Zeichen des Männlichen standen! Wir müssen freilich kritisch ins Feld führen, dass der argentinische Schriftsteller im Paratext zu seinem Roman Rayuela den männlichen Leser als den eigentlich kreativen und die weibliche Leserin als die im Grunde passive und bestenfalls an der Hand $\mathrm{zu}$ führende Leserin bezeichnete.

Sie können an der unglückseligen Metapher des „lector-hembra“ bei Cortázar unschwer erkennen, wie viel sich durch die Diskussionen der letzten Jahrzehnte in den Geschlechterbeziehungen verändert hat. Julio Cortázar hat seine Formulierung später bedauert, aber sie stand nun einmal in seinem Roman, und dort wollen wir sie auch nicht wieder herausschwindeln. Das Umdenken in den Geschlechterbeziehungen zeigte sich im Übrigen auch daran, dass ein ebenfalls in seinem Frühwerk noch von den Avantgarden mitgeprägter Italo Calvino - der freilich auch noch in manchen romanischen Traditionen der Geschlechterverhält- 
nisse stand - die ideale Leserfigur in seinem Se una notte d'inverno un viaggiatore weiblich ausstattete. Ich möchte freilich auf diesen Leseroman, der mit seiner starken Betonung der Legetik hervorragend in den Raum unserer Vorlesung gepasst hätte, hier nicht berücksichtigen, da ich mich Italo Calvino ausführlich in meinen Vorlesungen zu LiebeLesen gewidmet habe. ${ }^{2}$

Wir sollten uns davor hüten, die Worte von Romanfiguren oder Erzählerfiguren mit Aussagen der betroffenen realen Autoren gleichzusetzen, wie dies allzu oft selbst in der Forschungsliteratur erfolgt! Bei beiden Romanen spielen Geburt und Funktion des Lesers eine große und wichtige Rolle, ganz so, wie Roland Barthes dies in seinem Essay La mort de l'auteur von 1967 betont hatte. Steigen wir aber nun ein in einen dialogischen Monolog der Erzählerstimme in Kapitel 99 des Romans Rayuela:

Die Surrealisten glaubten, dass die wahrhaftige Sprache und die wahrhaftige Realität von der rationalistischen und bürgerlichen Struktur des Abendlandes zensiert und verdrängt waren. Sie hatten Recht, wie das jedweder Dichter weiß, aber dies war nicht mehr als ein Augenblick beim komplizierten Schälen der Banane. Das Ergebnis: Mehr als einer aß sie mitsamt der Schale auf. Die Surrealisten hingen sich an den Worten auf, anstatt sich brutal von ihnen zu lösen, wie es Morelli mit dem Wort selbst tun wollte. Fanatiker des Verbs im Reinzustand [...].

Doch rechnen wir Morelli nicht die Probleme von Dilthey, Husserl oder Wittgenstein zu. Das einzig Klare in all dem, was der Alte geschrieben hat, ist, dass wir, wenn wir weiterhin die Sprache mit ihrem geläufigen Schlüssel und mit ihren geläufigen Zielsetzungen benutzen, sterben werden, ohne jemals den wahren Namen des Tages erfahren zu haben. Es ist geradezu dumm zu wiederholen, dass sie uns, wie Malcolm Lowry sagte, das Leben verkaufen, und dass sie uns dieses vorgefertigt ausliefern. Auch Morelli insistiert geradezu dumm darauf, doch Etienne trifft den Nagel auf den Kopf: In der Praxis zeigt sich der Alte und zeigt uns den Ausweg. Wozu ist ein Schriftsteller denn nutze, außer um die Literatur zu zerstören? Und wir, die wir keine Leser-Weibchen sein wollen, wozu sind wir nutze außer dazu, bei dieser Zerstörung im Rahmen des Möglichen zu helfen? ${ }^{3}$

Die Problematik dieses Gesprächs zwischen Etienne und Oliveira über den abwesenden Morelli mündet ein in die Problematik eines Lesers, der sich aktiv an der Kunst, am Roman beteiligt und einen kreativen - wenn auch letztlich destruktiven - Part übernimmt. Wir bemerken, wie sehr dieser Text durch sprachphilosophische und allgemeinphilosophische Versatzstücke aufgeladen ist und zugleich zwei Einheiten in den Orkus zurückweist, die bislang wesentlich die kulturellen Traditionen geprägt hatten: das Bürgertum und das Abendland. Die

2 Vgl. hierzu Ette, Ottmar: LiebeLesen, S. 33-59.

3 Cortázar, Julio: Rayuela, S. 363. 
Lösung der Surrealisten aber, die Lösung der „destrucción“, wird nicht mehr als ausreichend empfunden. Sie kann nicht länger zur Lösung aller Probleme herangezogen werden. Und eben damit positioniert sich der Roman nach den historischen Avantgarden.

Das Thema der Zerstörung und Selbstzerstörung der Kunst hatten wir bei Antonin Artaud und bei Jusep Torres Campalans bereits im Kontext der historischen Avantgarden und ihrer Fluchtbewegungen aus Europa identifiziert. Jetzt erreicht es eine postsurrealistische Dimension mit der Fragestellung, wie denn diesen negativen Kategorien auf konstruktive Weise ein Ende zu bereiten sei. Die ästhetische Lösung, die Julio Cortázar immer wieder selbst auch praktizierte, war die einer Verstellung der Codes. Wir könnten an dieser Stelle auf den nur wenige Jahre später von Roland Barthes publizierten Text über Sade verweisen, in dem der Schriftsteller eben nicht länger als surrealistischer Zerstörer, sondern als kluger, aufmerksamer Sprachendieb dargestellt wird. ${ }^{4}$ Einer (avantgardistischen) Zerstörung allen Sprachmaterials wird bei weitem die Verstellung der Codes des literarischen Schreibens vorgezogen. An eben diesem Punkt siedelte sich die Kritik Cortázars an den historischen Avantgarden und ihren Ästhetiken des Bruchs an; und an demselben Punkt lässt sich auch seine eigene künstlerischliterarische Ästhetik verorten: Er hatte längst die Konsequenzen aus den Abenteuern der Avantgarden gezogen.

Volker Roloff hat vor einigen Jahren mit einigem Erfolg versucht, Cortázars Rayuela in die Tradition des Lektüreromans $\mathrm{zu}$ stellen. ${ }^{5}$ Er verwies darauf, dass schon Novalis betont hatte, ein Leser könne aus einem Buch machen, was er wolle. Genau dies solle Rayuela vorführen. Parallel zu dem wesentlich späteren Lektüreroman Se una notte d'inverno un viaggiatore von Italo Calvino zeige Cortázars Buch, dass viele Möglichkeiten narrativer Parcours und viele Schlüsse denkbar seien. Sie steckten aber stets in der Ambivalenz der Angebote, welche der Roman seiner Leserschaft macht und seien eben dadurch aufgehoben.

Es ist zweifellos schwierig, eine Nacherzählung des Cortázar'schen Textes zu bewerkstelligen, doch sollten wir dabei die romaneske Grundstruktur im Hinterkopf behalten, dass es sich auch im erzählerischen Sinne um ein Hüpfspiel handelt. Die zentrale Doppelfigur ist das Liebespaar Oliveira und seine Partnerin

\footnotetext{
4 Vgl. hierzu Ette, Ottmar: Der Schriftsteller als Sprachendieb. Versuch über Roland Barthes und die Philosophie. In: Nagl, Ludwig / Silverman, Hugh J. (Hg.): Textualität der Philosophie: Philosophie und Literatur. Wien - München: R. Oldenbourg Verlag 1994, S. 161-189.

5 Vgl. Roloff, Volker: Julio Cortázar: „Rayuela“. In (ders. / Wentzlaff-Eggebert, Harald, Hg.): Der lateinamerikanische Roman. Bd. II: Von Cortázar bis zur Gegenwart. Darmstadt: Wissenschaftliche Buchgesellschaft 1992, S. 78-90.
} 
La Maga, die im Paris der ausgehenden fünfziger Jahre zusammenleben, bis die Uruguayerin eines Tages spurlos verschwindet. Ab diesem Zeitpunkt verwandelt sie sich in das geradezu magische, mystisch überhöhte Ziel einer transzendenten Suche, die auf der anderen Seite des Ozeans, in einem argentinischen Irrenhaus, Oliveira obsessiv solange verfolgen wird, bis er in Talita, der Freundin Travelers, beim Hüpfspiel die verlorene Maga wiederzuerkennen glaubt.

Ich möchte Ihnen in der Folge zwei Auszüge aus dem Roman vorstellen, die für eine traditionelle, dem Roman linear folgende Leserschaft gleichsam Anfang und Ende des Buchs darstellen, also den Beginn von Kapitel 1 und das Ende von Kapitel 56. Nicht umsonst war Cortázar ein Meister der kurzen narrativen Form. Zwischen den beiden Kapiteln lassen sich viele mögliche Geschichten, viele „histoires“ mit ihren „récits“, unterbringen:

Würde ich die Maga wiederfinden? So viele Male hatte es mir genügt, von der Rue de Seine kommend unter dem Bogen zu erscheinen, der auf den Quai de Conti läuft, und das aschefarben-grünliche Licht, das auf dem Fluss lag, ließ mich kaum die Formen unterscheiden, und schon schrieb sich ihre schlanke Silhouette in den Pont des Arts ein, bisweilen von der einen auf die andere Seite wechselnd, bisweilen am eisernen Geländer stehen bleibend, über das Wasser gebeugt. Und es war so natürlich, die Straße zu überqueren, die Stufen zur Brücke hinaufzugehen, in deren schlanke Form einzutreten und mich der Maga zu nähern, die keineswegs überrascht lächelte, da sie wie ich davon überzeugt war, dass ein zufälliges Treffen das am wenigsten Zufällige in unseren Leben war, und dass die Leute, die sich genaue Rendezvous geben, dieselben sind, die auch ein liniertes Papier benötigen, um sich schreiben zu können, oder die von hinten eine Zahnpasta-Tube ausdrücken. ${ }^{6}$

Oliveiras Erinnerungen an die Maga sind unzertrennlich mit der Stadtlandschaft von Paris verknüpft, die als etwas gleichsam Natürliches erscheint und bei der Leserschaft fast selbstverständlich vorausgesetzt wird. Unverkennbar ist in dieser Passage das Motiv der Zufallsbegegnung zwischen zwei Liebenden mit der Idee des „hasard objectif“ verknüpft, des „objektiven Zufalls“. Damit wird die Dimension der Baudelaire’schen „modernité“, wie sie sich verbunden mit dem Motiv der Zufallsbegegnung in seinem berühmten Sonett $A$ une passante ausdrückte, insofern weiterentwickelt und fortgeschrieben, als es in dieser Passage eine Art kalkulierter Zufall, ja ein produzierter und von beiden in Szene gesetzter Zufall ist, welcher die Liebenden zusammenführt. Die Nähe zu André Breton und dessen Experimentaltext Nadja, den wir ja ebenfalls in unserer Vorlesung behandelt hatten, ist in dieser Begegnung mitten in der Pariser Stadtlandschaft ohne jede Frage gewollt. Cortázars Roman setzt so einiges bei seiner Leserschaft voraus -

6 Cortázar, Julio: Rayuela, S. 11. 
und dies nicht allein mit Blick auf das ansatzlose Auftauchen wie das Verschwinden Nadjas im surrealistischen Roman.

Zugleich dominiert von Beginn an die Frage und Suche nach etwas, was sich in den Namen „La Maga“ - fast eine Echowirkung des Namens „Nadja“ - packen lässt und mit dem die Leserschaft zunächst eher beiläufig eine der zentralen Figuren des Romans identifiziert, deren rätselhaftes Verschwinden dem Moment der Suche dann eine neue Dimension geben wird. Damit wird ein surrealistisches Versatzstück schon zu Beginn des Romans evoziert, wie auch insgesamt die Auseinandersetzung von Cortázars Roman mit dem Surrealismus von ganz besonderer Bedeutung ist: Auch das Schreibrezept des argentinischen Autors beruht darauf, ein wenig Avantgarde in mehr oder minder homöopathischen Dosen einzustreuen.

Der europäische Surrealismus wurde nicht nur für Autoren wie den Kubaner Alejo Carpentier und den guatemaltekischen Literaturnobelpreisträger Miguel Ângel Asturias zu einem Katalysator für das Erkennen der surrealen, der magischen Elemente der vielfältigen Kulturlandschaften Amerikas, sondern diente auch dazu, einen eigenständigen Blick auf den europäischen Surrealismus selbst zu werfen. Letzterer wurde einer grundlegenden Kritik unterzogen, die besonders auf die Dimension der „ruptura“, des Bruchs, abzielte. Insoweit lässt sich das Incipit von Cortázars Roman Rayuela, ausgerichtet am Motiv der Brücke entlang der Seine und damit aufgeladen durch die Dimension der sich im Fluss verkörpernden linearen, kontinuierlich ablaufenden Zeit und einer beide Ufer miteinander verbindenden Symbolik, verknüpfen mit der gesamten Problematik des Schreibens in einer Zeit nach den Avantgarden.

Denn dieses ZwischenWeltenSchreiben ${ }^{7}$ Julio Cortázars situiert sich nicht allein in einem postavantgardistischen Sinne zwischen den Avantgarden und ihren Neo-Avantgarden, sondern auch zwischen den beiden Welten Amerikas und Europas. Der Weg, der diese Suche ausmacht, ist jedoch nicht mehr einfachen hermeneutischen Bewegungsfiguren wie dem hermeneutischen Zirkel oder Kreis, Linie oder auch Stern zuordenbar, ${ }^{8}$ erweist sich doch in der Gesamtstruktur des Romans die Metaphorik des Weges durchbrochen von einem ständigen, diskontinuierlichen Hüpfspiel. Die dem Roman zu Grunde liegende Bewegungsfigur entzieht sich zumindest auf den ersten Blick zugleich jeder logischen Kausalität wie der kontinuierlichen Linearität und rückt vor allem den Konstruktionsge-

7 Vgl. Ette, Ottmar: ZwischenWeltenSchreiben. Literaturen ohne festen Wohnsitz (ÜberLebenswissen II). Berlin: Kulturverlag Kadmos 2005.

8 Vgl. Ette, Ottmar: ReiseSchreiben. Potsdamer Vorlesungen zur Reiseliteratur. Berlin - Boston: Walter de Gruyter 2020, S. 194-235. 
danken, die Konstruiertheit der eigenen Textualität von Beginn an ins Rampenlicht.

So haben wir es bei diesem Roman, um mit Arthur Rimbaud auf einen Präsurrealisten zurückzugreifen, durchaus mit einem „raisonné dérèglement de tous les sens“ zu tun, wenn wir mit „sens“ nicht nur die Sinne, sondern auch die Richtungen verstehen. Damit wird schon auf der Ebene der Bewegungen, welche stets Verstehensbewegungen der Lektüre anbahnen, die Ambivalenz, die Ambiguität, ja mehr noch die Vieldeutigkeit in den Roman eingeführt, welche sich schon im Oxymoron des kalkulierten Zufalls andeutete. Im Verhältnis dazu macht das Kapitel 56 seinerseits deutlich, dass auf Ebene der Story und nicht nur des Plot eine unauflösbare Ambiguität herrscht, die nicht in eine Eindeutigkeit überführt werden kann. Schon dies lässt erkennen, dass es - um die leicht differierende französische Begrifflichkeit zu wählen - nicht so sehr „histoire“ und „récit“ sind, für welche sich dieser Roman interessiert, sondern die „narration“, die Erzählweise und der Erzählprozess als solcher einerseits wie die Ebene des „discours“ und damit der Diskursivität andererseits.

Die Notizen des dritten Romanteils, die von den Aufschriften des Schriftstellers Morelli gebildet werden, sind also keineswegs verzichtbar, sondern essentieller Bestandteil eines Romans, der traditionellen Erzählschemata immer wieder explizit wie implizit den Kampf ansagt. Rayuela richtet sich also an Leserinnen und Leser, die wohl bestimmte Regeln befolgen, diese Regeln aber kreativ umzusetzen wissen. Doch hören wir uns zunächst noch das Ende jener Geschichte an, die der erste Leser, folglich der explizite Leser ersten Typs, mit Kapitel 56 abschließt:

\begin{abstract}
So war es, die Harmonie dauerte unglaublicherweise an, es gab keine Worte, um der Güte dieser beiden dort unten zu antworten, die ihn anschauten und vom Hüpfspiel aus zu ihm sprachen, denn Talita stand aufrecht und legte sich in Feld drei keine Rechenschaft davon ab, und Traveller hatte einen Fuß in Feld sechs, so dass das einzige, was er machen konnte, darin bestand, ein wenig die rechte Hand in einem schüchternen Gruß zu bewegen und weiter die Maga, Manú, anzublicken und sich dabei zu sagen, dass es schlussendlich ein Wiedersehen gab, auch wenn es nicht länger als diesen grässlich süßen Augenblick lang dauern konnte, in dem es ohne Zweifel das Beste gewesen wäre, sich ein wenig nach außen zu beugen und sich gehen zu lassen, paff und Schluss. ${ }^{9}$
\end{abstract}

Die zum vorigen Zitat symmetrische Anlage und damit zum Incipit des gesamten Romans ist offenkundig und bis in die zentralen Lexeme der Passage nachvollziehbar. In dem vor Oliveiras Fenster in der Irrenanstalt von den Erwachsenen

9 Cortázar, Julio: Rayuela, S. $284 \mathrm{f}$. 
Traveler und Talita gespielten Kinder- und Hüpfspiel von Himmel und Hölle überlagern sich die narrativen Strukturen von Teil 1 („Del lado de allá“) und Teil 2 („Del lado de acá“). Dabei bleibt allerdings unklar, ob Talita wirklich die Maga ist und Oliveira am Ziel und Ende seiner Suche sich wirklich aus dem Fenster seiner Anstalt in Argentinien stürzt. Denn er neigt sich ganz so nach außen, wie sich die Maga einst in seinen Augen über das Geländer des Pont des Arts zu Paris gebeugt hatte. Ja, es war zum „encuentro“, zum Treffen und Wiedersehen, gekommen: Am Ende steht eine Antwort auf die Eingangsfrage des Romans. Und doch bleibt alles radikal offen und vieldeutig. Umso wichtiger ist angesichts dieser unauflösbaren Polysemie die Rolle der Leserschaft, die für sich Entscheidungen zu treffen hat gerade in Bezug auf das Buch, mithin auf die beiden oder die vielen Bücher, welche die Leserinnen und Leser in Gestalt aller Kapitel vor sich haben. Julio Cortázars Roman, so könnten wir sagen, wird zu einem Konstrukt seiner Leserinnen und Leser selbst: Die Poetik des Romans ist seine Legetik.

Cortázars Schriftsteller Morelli möchte aus dem Leser einen Komplizen und zugleich kreativen und produktiven Weggefährten machen. Rayuela realisiert dieses Konzept ganz so, wie der Roman von Max Aub manche Vorstellungen von Jusep Torres Campalans bereits umsetzte. Es ließe sich daher sagen, dass der Figur des realen textexternen Autors jeweils die Figur eines fiktiven textinternen Künstlers spiegelsymmetrisch entspricht und so ein dialogales Verhältnis zwischen beiden Figuren entsteht, die freilich nicht miteinander gleichgesetzt werden dürfen. Der offene und unabschließbare Prozess der Lektüre wird in Rayuela sehr weit vorangetrieben und gerinnt zu einem Konstruktionsmerkmal des Romans selbst. Er wird zu einem schöpferischen und zu eigenen Schöpfungen aufrufenden Angebot an den Leser, sind doch auch andere Parcours als die beiden vom eingangs positionierten „Tablero“ vorgezeichneten möglich. Ohne das aktive und interaktive Mitspielen der Leserschaft bliebe der Roman gänzlich abstrakt und wie ein niemals gespieltes Theaterstück - in seiner reinen Virtualität gefangen. Rayuela bietet auf diese Weise ein Schreibmodell, das sich nicht nur für literarische Texte zur Nachahmung und Weiterentwicklung anbietet.

Die Leserinnen und Leser aber müssen den Text in bestimmter Weise aufführen - und gerade dieser Zwang zur Entscheidung ermöglicht es dem Roman, die Leserschaft zu einer Parteinahme, einer kreativen Entscheidung zu zwingen. Aus dieser Perspektive ist Rayuela Roman und zugleich Partitur für vielfältige Aufführungen. In diesem Zusammenhang ist es auch nicht unwesentlich, dass die Erzählerfigur in der Tat in eine Reihe von Einzelpositionen zerfallen ist und mit ihren diversen Blickpunkten keine innere Kohärenz mehr aufweist. Auch an dieser Stelle werden wir wieder an den Satz von Roland Barthes erinnert, dass die Geburt des Lesers mit dem Tod des Autors (und vielleicht eben auch einer kohärenten Erzählerfigur) bezahlt werden muss. Rayuela liest sich in dieser Hinsicht 
durchaus als kubistischer Roman im Sinne Max Aubs beziehungsweise Jusep Torres Campalans'.

Auf all seinen narrativen und diskursiven Ebenen ist Julio Cortázars Romanwerk selbstreferentiell und integriert auf fundamentale Weise die Metaliteratur in die Literatur selbst. Die Theorie des Romans wird zum Roman der Theorie sicherlich bei Cortázar kein neuartiges Verfahren, aber doch eines, das mit großer Kreativität und Radikalität aufzuwarten versteht. Die Vielzahl an Formen und Figuren der Autoreferentialität können an dieser Stelle nicht ausgeführt werden, sollten aber eingehen in unser Bild eines Romans, der wohl wie kein anderer die Phase konstruktiver Vorbereitung eines romanhaften Schreibens im Zeichen der Postmoderne verkörpert. Die Vorbereitung des Romans, „La Préparation du roman“, ist - um erneut mit Roland Barthes zu sprechen - zum Roman selbst geworden.

Wir haben mit diesem in den fünfziger Jahren angelegten und erstmals 1963 veröffentlichten umfangreichen Romanwerk des argentinischen Schriftstellers eine Phase erreicht, in der - zu Beginn der sechziger Jahre - die Romanentwicklung einen Zeitraum abschloss, welchen man noch als „en amont“ der poststrukturalistischen beziehungsweise postmodernen Theoriebildungen im engeren Sinne bezeichnen könnte. Ich möchte an dieser Stelle nicht mehr versuchen, die Ergebnisse unseres Parcours noch einmal zusammenzufassen und bin überzeugt davon, dass sich bereits jetzt die Konturen eines Schreibens im Zeichen der Postmoderne herausgeschält haben. Anhand der in unserer Vorlesung behandelten Reihe von Texten, ausgehend von Jorge Luis Borges, vor allem aber dann auch mit Boris Vian, Max Aub und nicht zuletzt Julio Cortázar, haben wir bereits eine zentrale Erfahrung gemacht. Sie beinhaltet die Erkenntnis, dass sich die Schreibformen im Zeichen der Postmoderne in ganz grundlegender Weise als eine Art produktiver Auseinandersetzung mit den Schreibformen und Ästhetiken der historischen Avantgarden und insbesondere des Surrealismus entwickelt und herausgebildet haben. Dieser Befund ist für die herkömmliche Sichtweise der literarhistorischen wie literarästhetischen Entwicklungen im Verlauf des 20. Jahrhunderts durchaus bemerkenswert, ja erstaunlich.

Gerade die produktive Aneignung einer Reihe avantgardistischer Verfahren im Kontext einer nicht länger auf Schock, auf Infragestellung der Institution Kunst ausgelegten „écriture“ und Ästhetik sollte deutlich gemacht haben, dass es nicht immer leicht ist, die Postmoderne selbst von den Avantgarden im Detail abzutrennen, wenn uns dies auch auf theoretischer Ebene einfacher gelingen mag. Vor allem aber möchte ich bei Ihnen die Einsicht in den Sachverhalt fördern, dass es sehr gute Gründe dafür gibt, warum entscheidende Impulse für die Entfaltung von Ästhetiken im Zeichen der Postmoderne gerade aus den Literaturen Lateinamerikas kamen. Denn letztere bildeten von Beginn an Sichtweisen eines avant- 
gardistischen Kunstverständnisses heraus, die nichts mit Schock, Bruch und Zerstörung zu tun hatten. So darf man sehr wohl der Überzeugung Ausdruck geben, dass der entscheidende Anstoß zur Ausprägung von Ästhetiken im Zeichen der Postmoderne eben aus Lateinamerika kam. Die ,Neue Welt 'ist folglich für eine adäquate Sichtweise auch und gerade der europäischen, also altweltlichen Literaturen aus heutigem Blickwinkel unverzichtbar.

Im weiteren Verlauf unserer Vorlesung, die den sechziger, siebziger und achtziger Jahren gewidmet sein wird, soll nicht zuletzt kritisch erfasst werden, ob und inwiefern sich in den neunziger Jahren und zu Beginn des 21. Jahrhunderts eine neue Ästhetik Gehör zu verschaffen begann, welche sich deutlich nach der Postmoderne positionierte. Die folgenden Analysen und Überlegungen könnten uns aufzeigen, wie das Schreiben im Zeichen der Postmoderne in einem wesentlich direkteren Polylog mit den unterschiedlichen Literaturen der Welt überging. Die intertextuellen Dialoge mit Philosophie und Denkformen von Poststrukturalismus, „Déconstruction“ und „Deconstruction“ leiteten im Kontext einer verschärften Massenkommunikation im weltweiten Maßstab über zu neuen Verfahren und Formen, welche nur scheinbar zu einem Schreiben nach der sogenannten ,terreur théorique“ führten, wurden die großen Theorie-Debatten doch jetzt nicht mehr von Paris aus angezettelt. Doch ich muss aufpassen, den nachfolgenden Sitzungen nichts vorwegzunehmen: Versuchen wir also, den Faden unserer Argumentation im transatlantischen Spiel wieder auf der Seite Frankreichs aufzunehmen! 